Research Article

\title{
Employment of Alginate Floating In Situ Gel for Controlled Delivery of Celecoxib: Solubilization and Formulation Studies
}

\author{
Bazigha K. Abdul Rasool ${ }^{(D,}{ }^{1}$ AlZahraa Khalifa, ${ }^{1}$ Eman Abu-Gharbieh $\left(\mathbb{D}^{2},{ }^{2}\right.$ and Rawoof Khan ${ }^{3}$ \\ ${ }^{1}$ Pharmaceutics Department, Dubai Pharmacy College for Girls, Dubai, UAE \\ ${ }^{2}$ Clinical Sciences Department, College of Medicine, University of Sharjah, Sharjah, UAE \\ ${ }^{3}$ Dubai Institute for Environmental Research and Laboratory Analysis, Dubai, UAE
}

Correspondence should be addressed to Bazigha K. Abdul Rasool; bazigha@dpc.edu

and Eman Abu-Gharbieh; eabugharbieh@sharjah.ac.ae

Received 26 March 2020; Revised 25 April 2020; Accepted 2 May 2020; Published 1 June 2020

Academic Editor: Ali Nokhodchi

Copyright ( 2020 Bazigha K. Abdul Rasool et al. This is an open access article distributed under the Creative Commons Attribution License, which permits unrestricted use, distribution, and reproduction in any medium, provided the original work is properly cited.

Celecoxib $(\mathrm{CXB})$ is a COX-2-selective nonsteroidal anti-inflammatory drug used to control pain and various inflammatory conditions. CXB has limited oral bioavailability and a slow dissociation rate due to its poor water solubility. In order to enhance the oral bioavailability of $\mathrm{CXB}$ and reduce the frequency of administration, the present study was aimed at enhancing the aqueous solubility of $\mathrm{CXB}$ by a cosolvency technique and then at formulating and evaluating a CXB in situ floating gelling system for sustained oral delivery. Three cosolvents, namely, PEG 600, propylene glycol, and glycerin, at different concentrations, were used to solubilize CXB. Particle size analysis was performed to confirm the solubility of CXB in the solutions. The floating in situ gel formulations were then prepared by the incorporation of the CXB solution into sodium alginate solutions $(0.25,0.5$, and $1 \% \mathrm{w} / v)$. Formulations, in sol form, were then in vitro characterized for their physical appearance, $\mathrm{pH}$, and rheological behaviors, while formulations in gel form were evaluated for their floating behavior and in vitro drug release studies. FTIR spectroscopy was performed to examine drug-polymer interaction. The selected formula was evaluated biologically for its anti-inflammatory and analgesic activities. Results revealed that the less-polar solvent PEG 600 at $80 \% v / v$ had the highest solubilization potential, and it was used to optimize the in situ gel formulation. The candidate formula (F3) was found to have the highest sodium alginate concentration $(1 \% \mathrm{w} / v)$ and showed the optimum sustained release profile with the Higuchi model release kinetics. The results from the FTIR spectroscopy analysis showed noticeable drug-polymer molecular interaction. Moreover, F3 exhibited a significantly higher percentage of paw edema inhibition at $8 \mathrm{~h}$ compared with the reference drug $(p<0.05)$. Also, it showed a sustained duration of analgesia that persisted for the entire experimental time.

\section{Introduction}

Celecoxib (CXB), a selective COX-2 inhibitor, is a nonsteroidal anti-inflammatory drug used primarily for the treatment of various inflammatory and painful conditions [1]. Currently, the available dosage forms of CXB are intended only for oral administration. However, the oral absorption of CXB is limited with a bioavailability ranging from 22 to 40\% [2]. According to the Biopharmaceutics Classification System (BCS), CXB is classified as a Class II drug that is practically insoluble at $\mathrm{pH}$ conditions in the gastrointestinal tract [3]. The extremely low water solubility of CXB is related to many formulation problems and limits its therapeutic effi- ciency by delaying the rate of absorption and onset of action. Therefore, it is essential to improve its solubility as it is a potential candidate for advanced solubilization techniques.

The literature reported various solubilizing techniques to improve the solubility and oral absorption of CXB, such as a self-emulsifying system [4], mesoporous silica [5], nanosuspension [6, 7], elixir [8], and solid dispersion [9]. Nevertheless, in previous studies, no attempt has been taken to investigate the aqueous solubility enhancement of CXB by the cosolvency approach.

On the other hand, due to several variables that change throughout the gastrointestinal tract which significantly affect drug absorption, it is evident that the conventional 
drug delivery system does not easily overcome the difficulties imposed by the gastrointestinal system.

For instance, the incomplete dissolution of the drug and the associated reduction of dose effectiveness are consequences of the incapacity of the conventional drug delivery system to be retained at the stomach level [10]. Therefore, there is a need to develop a sustained release dosage form that can improve the oral bioavailability of CXB and also reduce repeated administrations.

In order to overcome these challenges, technological researchers have developed pharmaceutical systems that control drug release and the residence time, some of which are already available on the market. Gastroretentive drug delivery systems are a good example; they emerged to enhance the bioavailability and effectiveness of drugs with a narrow absorption window.

The in situ gelling systems have become more distinguished among the various novel drug delivery systems. These are polymeric formulations that are in sol forms before entering the body but change to gel forms under the physiological conditions such as $\mathrm{pH}$ change, temperature modulation, or solvent exchange [11].

Numerous advantages of the in situ gelling system, such as stability, biocompatibility, reproducibility, ease of application, and reduced frequency of administration, make the in situ gel dosage form more attractive for commercial use [11]. Moreover, the in situ gel dosage form would improve patient compliance and comfort, exhibit sustained and controlled rates of absorption and excretion, and possess a reasonable margin of safety [12].

Various natural and synthetic polymers are currently used for oral, buccal, rectal, vaginal, ocular, intraperitoneal, and parenteral in situ gel formulation. Pectin, xyloglucan, gellan gum, chitosan, and alginic acid are some of the natural polymers [13].

This study is aimed at developing a CXB in situ gel system for sustained oral drug delivery using natural biodegradable polymers and the cosolvency technique to enhance the aqueous solubility of $\mathrm{CXB}$ and at evaluating the bioactivity of the prepared formula on animal models.

\section{Materials and Methods}

2.1. Materials. CXB BP was purchased from Sigma-Aldrich, USA. Polyethylene glycol (PEG 600), propylene glycol (PG), and glycerin were from Alpha Chemika, India. Sodium alginate (SA) (Fluka) $90 \mathrm{cps}(1 \% \mathrm{w} / v$ solution in water at $\left.25^{\circ} \mathrm{C}\right)$ and calcium chloride $\left(\mathrm{CaCl}_{2}\right)$ (E. Merck, India) were used. All other reagents were of analytical grade.

2.2. Estimation of CXB. CXB estimation was performed spectrophotometrically at $\lambda_{\max }$ of $252 \mathrm{~nm}$ using a UV-1800 UVVIS spectrophotometer (Shimadzu, Japan). The stock solution was prepared in methanol at a $1000 \mu \mathrm{g} / \mathrm{mL}$ strength, and the drug's absorbance was recorded against the blank, methanol. The calibration curve was prepared at concentration ranges between 6 and $24 \mu \mathrm{g} / \mathrm{mL}$ to estimate the drug content [14]. The method was validated for linearity, accuracy, and precision. The experiments were repeated three
TABLE 1: Composition of CXB floating in situ gel formulations.

\begin{tabular}{lccc}
\hline Ingredients $(\% w / v)$ & F1 & F2 & F3 \\
\hline CXB & 0.6 & 0.6 & 0.6 \\
SA & 0.25 & 0.5 & 1.0 \\
Calcium chloride & 0.15 & 0.15 & 0.15 \\
Calcium carbonate & 0.5 & 0.5 & 0.5 \\
Citric acid & 0.1 & 0.1 & 0.1 \\
Sodium citrate & 1.0 & 1.0 & 1.0 \\
\hline
\end{tabular}

times to check its reproducibility. CXB absorbance was measured as a mean $\pm \mathrm{SD}(n=3)$. The regression equation for the calibration curve was generated from the linear regression analysis on a Microsoft Excel worksheet 2010 and was later used for the determination of CXB concentration.

2.3. Solubility Studies. Excess amount of CXB pure powder was added to mixtures of distilled water and cosolvents (PEG 600, PG, and glycerin) at various concentrations (0, $20,40,60,80$, and $100 \% v / v)$. The mixtures were placed in conical flasks and closed well with stoppers. The flasks were shaken using a Thermo Scientific Precision SWB-15 shaking water bath at a shaking speed of $50 \mathrm{rpm}$ and temperature of $25 \pm 0.5^{\circ} \mathrm{C}$ for $48 \mathrm{~h}$ to obtain equilibrium [15]. Then, aliquots were withdrawn, filtered through a Millipore ${ }^{\circledR}$ filter membrane $(0.45 \mu \mathrm{m}$ pore size $)$, and diluted appropriately. The samples were analyzed using a UV-1800 UV-VIS spectrophotometer (Shimadzu, Japan) at $252 \mathrm{~nm}$ wavelength, and the solubility of $\mathrm{CXB}(\mathrm{mg} / \mathrm{mL})$ in each sample was determined.

2.4. Particle Size of CXB-Cosolvent Mixture. The particle size was measured in a range of $0.1 \mathrm{~nm}$ and $10 \mu \mathrm{m}$ by using a Malvern $^{\circledR}$ Zetasizer. Particle size measurement was used at the point of maximum solubility. One millilitre of each sample was diluted up to $10 \mathrm{~mL}$ with distilled water $(1: 10 \mathrm{v} / \mathrm{v})$ and filtered through a Millipore ${ }^{\circledR}$ membrane filter (pore size $0.45 \mu \mathrm{m})$. Samples were then placed in a capillary measurement cell, and particle size analysis was carried out at $25^{\circ} \mathrm{C}$.

2.5. Preparation of CXB In Situ Floating Gel Formulations. Three formulas of the CXB in situ floating gel were prepared by dispersing the CXB powder thoroughly in SA solutions $(0.25 \%, 0.5 \%$, and $1 \% w / v)$. SA aqueous solutions were prepared by heating to $60^{\circ} \mathrm{C}$ with continuous stirring followed by cooling to $40^{\circ} \mathrm{C}$. Then, $\mathrm{CaCl}_{2}$ (cross-linking agent), $\mathrm{CaCO}_{3}$ (gas-forming agent), citric acid, and sodium citrate were added to the mixture. The composition of CXB floating in situ gel formulations is shown in Table 1.

\subsection{In Vitro Characterization of CXB In Situ Gels}

2.6.1. Physical Appearance and $p H$. The color and the clarity of the floating in situ gel formulations were evaluated by the visual inspection of the sols against a dark and white illuminating background. The $\mathrm{pH}$ of the formulas was measured in triplicate using an Edge HI2002-01 pH meter (Hanna Instruments) at room temperature. 


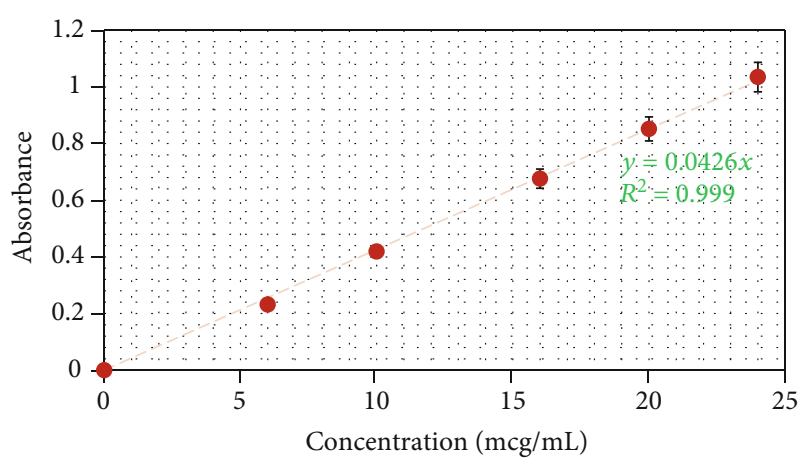

Figure 1: Calibration curve of $\mathrm{CXB}$ in methanol (mean $\pm \mathrm{SD}, n=3$ ).

2.6.2. Rheological Behavior. The prepared formulas were placed inside a sample adaptor of a Brookfield DV-III Ultra Rheometer. The rheological behavior of the gel formulations was examined at different angular velocities $(10,20,30,50$, 60 , and $100 \mathrm{rpm}$ ) by using a spindle no. S27. The viscosity was determined at $25^{\circ} \mathrm{C}$, as a mean $\pm \mathrm{SD}$ of triplicate measurements.

2.6.3. Floating Behavior. The floating behavior was determined by observing the floating lag time (FLT), the time taken for the formula to emerge to the surface of the medium, and the duration of floating (DOF), the time the formula constantly floated on the medium surface for each formula. The in vitro gelling capacity was graded in three categories based on FLT and DOF [15], as follows:

Low gelling capacity (+): FLT (immediate gelation) and $\mathrm{DOF}<12 \mathrm{~h}$

Intermediate gelling capacity (++): FLT (immediate gelation) and $24 \mathrm{~h}>\mathrm{DOF}>12 \mathrm{~h}$

High gelling capacity (+++): FLT (immediate gelation) and DOF $>24 \mathrm{~h}$

This study was performed by placing an accurately weighed sample $(1 \mathrm{~g})$ of each formula in a test tube containing $10 \mathrm{~mL}$ of the simulated gastric fluid USP without pepsin enzyme (constituents: $2 \mathrm{~g} \mathrm{NaCl}$ and $7 \mathrm{~mL} \mathrm{HCl}$ in D.W. to make $1 \mathrm{~L}$ ), and samples were then observed for their floating behavior.

2.7. In Vitro Drug Release. The in vitro release of CXB from the formulations was determined using a USP dissolution apparatus type II (paddle). The vessels were filled with $900 \mathrm{~mL}$ of the dissolution medium $(0.1 \mathrm{~N} \mathrm{HCl}, \mathrm{pH} 1.2)$. The temperature was maintained at $37 \pm 0.5^{\circ} \mathrm{C}$, and the operating speed was $50 \mathrm{rpm}$ [16]. Samples of $5 \mathrm{~mL}$ were withdrawn at $0.5,1,2,3,4,5,6,7$, and $8 \mathrm{~h}$ and replaced with the same volume of the fresh buffer to maintain the sink condition. The drug content in samples was determined as a cumulative percentage release by using a UV-1800 UV-VIS spectrophotometer (Shimadzu, Japan) at $252 \mathrm{~nm}$.

To understand the mechanism of CXB release from the in situ gels, the in vitro dissolution data of the prepared formulas were fitted to different kinetics models, including firstorder kinetics, zero-order kinetics, the Higuchi model, and the Korsmeyer-Peppas model [17]. The release rate constants

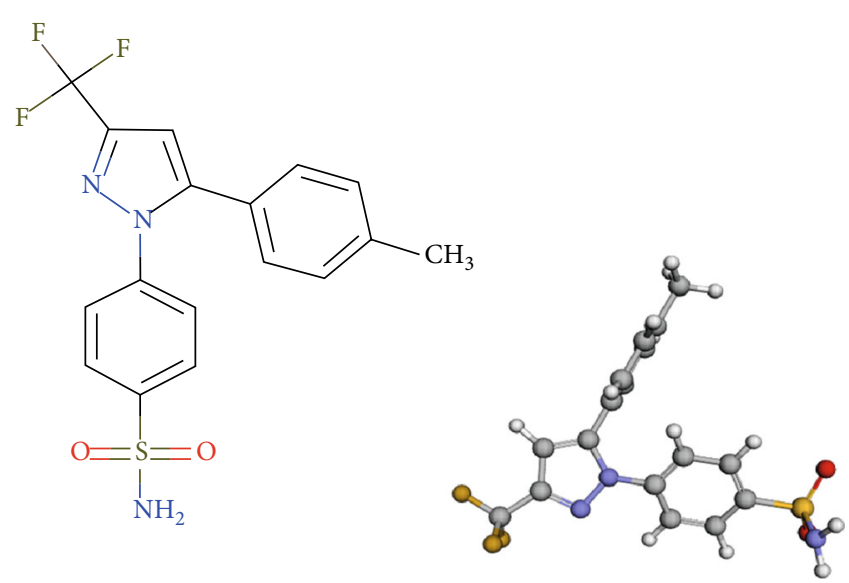

Figure 2: Chemical structure for CXB.

and correlation coefficients $\left(R^{2}\right)$ were obtained from the linear regression analysis on Microsoft ${ }^{\circledR}$ Excel 2013.

2.8. FTIR Spectroscopy Analysis. Samples $(10 \mathrm{mg})$ of CXB powder, SA powder, $\mathrm{CXB} / \mathrm{SA}$ physical mixture, and freezedried gel were loaded into a Shimadzu FTIR spectroscope (IRAffinity-15, Japan), and their spectra were recorded over a wave range of $450-4000 \mathrm{~cm}^{-1}$. The CXB-loaded SA gel was dried by a Biobase Freeze Dryer (BK-FD10P, China) under the conditions of $-59^{\circ} \mathrm{C}$ and $0.001 \mathrm{mp}$ vacuum pressure for $24 \mathrm{~h}$.

The CXB/SA physical mixture was prepared by continuous mixing in a mortar and pestle of CXB with the polymer at a molar ratio of $1: 1 \mathrm{w} / \mathrm{w}$ for $30 \mathrm{~min}$. FTIR spectroscopy analysis was performed to study the possibility of the molecular interaction between CXB and SA.

\subsection{Biological Evaluation}

2.9.1. Experimental Animals. Thirty-six adult, healthy Wistar albino rats of both sexes weighing $150 \mathrm{~g}$-200 g, approximately two months old, were used for the biological evaluation studies. The animals were maintained under standard conditions, fed with regular diet and water supplied ad libitum, and accommodated for seven days before the experiments. The experimental protocol followed the ethical standards for laboratory animals [18] and was approved by the Ethical Research Committee of Dubai Pharmacy College, Dubai, United Arab Emirates.

2.9.2. Experimental Protocol. Based on the in vitro results, the best formula was selected to proceed to the in vivo evaluation. Animals were randomly divided into six groups of six animals each. The first three groups were used for the antiinflammatory activity evaluation, and the others were used for the analgesic test.

In each experiment, the first group of animals served as the control and received oral saline. The second group served as a positive control group and received a $50 \mathrm{mg} / \mathrm{kg}$ dose of the reference drug, Celebrex ${ }^{\circledR}$ capsules, orally, and the third group received the F3 formula orally at a dose of 25 and $50 \mathrm{mg} / \mathrm{kg}$. 
TABLE 2: Solubility profile of CXB in water-PEG 600, PG, and glycerol mixtures at $25^{\circ} \mathrm{C}$.

\begin{tabular}{|c|c|c|c|c|c|c|c|}
\hline \multirow{2}{*}{ Water $(\% v / v)$} & \multirow{2}{*}{ Cosolvent $(\% v / v)$} & \multicolumn{2}{|c|}{ PEG 600} & \multicolumn{2}{|r|}{ PG } & \multicolumn{2}{|c|}{ Glycerol } \\
\hline & & DC & $\mathrm{CXB}(\mathrm{mg} / \mathrm{mL})$ & DC & $\mathrm{CXB}(\mathrm{mg} / \mathrm{mL})$ & DC & $\mathrm{CXB}(\mathrm{mg} / \mathrm{mL})$ \\
\hline 0 & 100 & 11.60 & $2.898 \pm 0.0828$ & 32.00 & $1.860 \pm 0.0448$ & 42.50 & $0.395 \pm 0.0196$ \\
\hline 20 & 80 & 24.95 & $3.044 \pm 0.0552$ & 41.27 & $1.946 \pm 0.0383$ & 49.67 & $0.406 \pm 0.0153$ \\
\hline 40 & 60 & 38.30 & $0.741 \pm 0.0312$ & 41.27 & $0.637 \pm 0.0175$ & 56.84 & $0.265 \pm 0.0072$ \\
\hline 60 & 40 & 51.66 & $0.177 \pm 0.0098$ & 50.54 & $0.164 \pm 0.0072$ & 64.02 & $0.112 \pm 0.0048$ \\
\hline 80 & 20 & 65.00 & $0.045 \pm 0.0043$ & 95.82 & $0.036 \pm 0.0032$ & 71.19 & $0.059 \pm 0.0032$ \\
\hline 100 & 0 & 78.38 & $0.0022 \pm 0.002$ & 78.36 & $0.002 \pm 0.0018$ & 78.36 & $0.002 \pm 0.0032$ \\
\hline
\end{tabular}

DC: dielectric constant $(\varepsilon)$; results are given as mean \pm S.D. $(n=3)$.

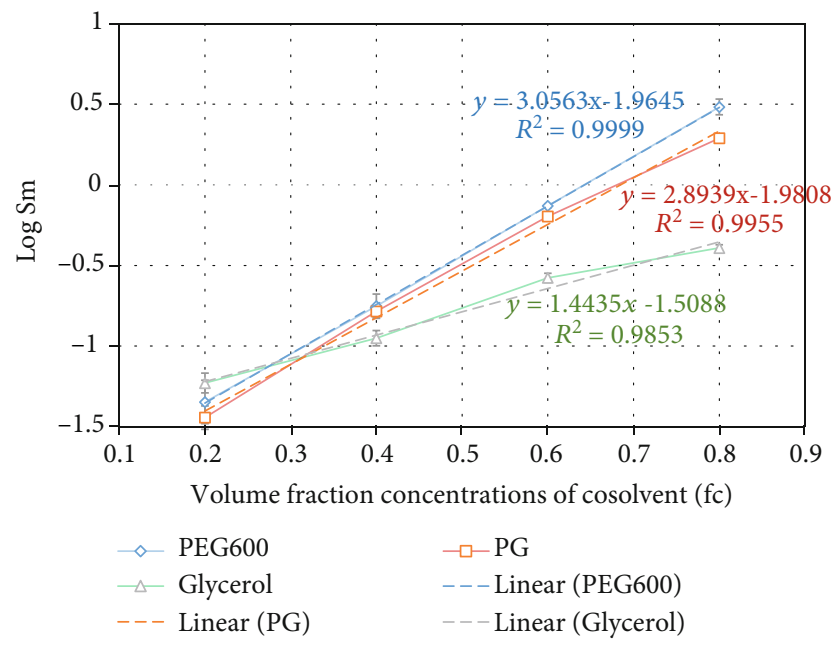

Figure 3: Log-linear solubilization plot of CXB in different cosolvent mixtures (mean $\pm \mathrm{SD}, n=3$ ).

2.9.3. Anti-Inflammatory Activity. Carrageenan-induced paw edema in the rats' test was used to assess the acute antiinflammatory activity of the developed formula. One-hour posttreatment, the inflammation was induced by injection of $0.1 \mathrm{~mL}$ of $1 \%(w / v)$ carrageenan solution into the left hind paw of each rat. The baseline paw volumes of all animals were recorded using a LE7500-digital plethysmometer (Harvard, USA). The relative increase in paw volume was determined by measuring the paw volume after $1,3,5$, and $8 \mathrm{~h}$, following the carrageenan administration [19]. The percentage inhibition of edema was compared with the controls. The percentages of inhibition were obtained using the following formula:

$$
\begin{aligned}
& \text { Percentage of inhibition } \\
& \qquad=\frac{\left(V_{t}-V_{0}\right) \text { control }-\left(V_{t}-V_{0}\right) \text { treated }}{\left(V_{t}-V_{0}\right) \text { control }} \times 100,
\end{aligned}
$$

where $V_{t}$ and $V_{0}$ are the average volumes for each group posttreatment and pretreatment, respectively.

2.9.4. Analgesic Effect. Thirty minutes before treatment, $1 \%$ $w / v$ carrageenan suspension $(0.01 \mathrm{~mL})$ was injected into the supplanter area of the right hind paw of the animal. In the test, the rat was placed on a hot plate maintained at $55 \pm 0$. $5^{\circ} \mathrm{C}$. The time elapsed until the occurrence of either a hind paw licking or a jump off the surface was recorded as the hot plate latency. The animals were tested at $0,0.5,1.0$, and $3.0 \mathrm{~h}$. The cut-off time was $50 \mathrm{~s}$ to prevent tissue damage, and mice with baseline latencies of $<5$ were eliminated from the study [20].

2.10. Statistical Analysis. The measurements were expressed as mean values along with their standard deviations. For the in vitro studies, the statistical assessment was conducted using the analysis of variance followed by Bonferroni's correction for multiple comparisons. A $p$ value $<0.05$ was considered significant. Calculations were performed using GraphPad Prism Software Version 6.

\section{Results and Discussion}

3.1. CXB Calibration Curve. CXB calibration curve was prepared by plotting the mean absorbance $\pm \mathrm{SD}$ of $\mathrm{CXB}$ in the diluted solutions against their relevant concentrations (Figure 1). Linear regression of absorbance on concentrations gave the equation $y=0.0426 x$ with a correlation coefficient $\left(R^{2}\right)$ of 0.999 . The high value of $R^{2}$ indicated that the concentrations used in the preparation of the calibration curve were convenient and in compliance with Beer's law.

3.2. Solubility of CXB. Basically, the addition of a cosolvent is an effective technique to enhance the solubility of poorly soluble drugs [21, 22]. Cosolvents are water-miscible solvents, commonly used in pharmaceutical manufacturing to enhance drug solubilization. The nonpolar hydrocarbon region within the cosolvent reduces the ability of the aqueous system to repel nonpolar solutes. In the present study, three widely used cosolvents, PEG 600, PG, and glycerol, were evaluated for the aqueous solubility of CXB. The cosolvent with higher drug solubility in the pure state is referred to be the stronger solvent.

CXB has a very poor solubility in water that is attributed to a predominantly nonpolar feature of its molecule structure (Figure 2). The solubility of CXB in various water-cosolvent mixtures with their respective dielectric constants is represented in Table 2. Results indicated an inverse relationship between CXB solubility and solvent polarity. The cosolvent efficiency ratio, i.e., the ratio of solubility of a drug in a cosolvent-water mixture to the solubility of the drug in water without PEG 600, PG, and glycerol $(80 \% v / v)$, was found to 


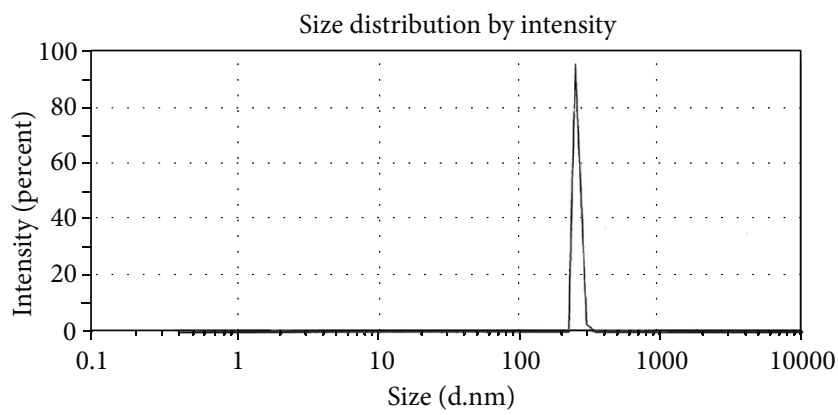

FIgURe 4: Particle size distribution curve by the intensity of CXB in PEG $60080 \% v / v$.

TABLE 3: $\mathrm{pH}$ value and floating behavior of CXB in situ gels*.

\begin{tabular}{lccc}
\hline Formulation code & F1 & F2 & F3 \\
\hline pH & $5.65 \pm 0.106$ & $6.14 \pm 0.035$ & $6.21 \pm 0.064$ \\
FLT $(\mathrm{sec})$ & $8.32 \pm 0.009$ & $9.34 \pm 0.047$ & $22.5 \pm 0.201$ \\
DOF (h) & $>24$ & $>24$ & $24 \mathrm{~h}>\mathrm{DOF}>12 \mathrm{~h}$ \\
Gelation capacity & +++ & +++ & ++ \\
\hline
\end{tabular}

${ }^{*}$ Results are presented as mean $\pm \mathrm{SD}(n=3)$.

be $1383.6,884.5$, and 184.5 , respectively. This is probably because of extensive hydrophobic interactions between the drug and the solvent.

The dielectric constants of the solvent mixtures were calculated by the following formula:

$$
\varepsilon_{\mathrm{mix}}=\varepsilon_{\mathrm{ws}} f_{\mathrm{ws}}+\varepsilon_{\mathrm{ss}} f_{\mathrm{ss}}
$$

where $\varepsilon$ and $f$ are the dielectric constant and volume fraction, respectively, and the subscripts mix, ws, and ss represent values for the mixture, weaker solvent, and stronger solvent, respectively.

Solubility usually increases with a decrease in the dielectric constant of the mixture. However, a considerable reduction in CXB solubility was achieved by using the cosolvents alone compared with $80 \% v / v$ of cosolvent-water mixtures. This effect occurs because $\mathrm{CXB}$ has some degree of polar property as well and maximum solubilization related to the polarity of the solute and the solvent.

In addition, the solubility in a water-cosolvent mixture (Sm) was calculated from the solubility values in pure water $(\mathrm{Sw})$ and in the neat cosolvent $(\mathrm{Sc})$ and the volume fraction concentrations of water and the cosolvent, fw and fc, respectively, in the solvent mixture

$$
\log \mathrm{Sm}=\mathrm{fc} \log \mathrm{Sc}+\mathrm{fw} \log \mathrm{Sw}
$$

Therefore, the solubilization of hydrophobic drugs in water-cosolvent mixtures was represented by the log-linear model [23] that is expressed sometimes using the solubilization power of the cosolvent $(\sigma)$ as follows:

$$
\log \mathrm{Sm}=\log \mathrm{Sw}+\sigma \mathrm{fc}
$$

where $\sigma=\log (\mathrm{Sc} / \mathrm{Sw})$ is the solubilization power of the cosolvent.

The solubility data of CXB in the water-cosolvent mixtures were used to plot a log-linear solubilization curve, as shown in Figure 3. Results revealed that the maximum cosolvent solubilizing effect of the three cosolvents was obtained at the concentration of $80 \% v / v$. PEG $60080 \% v / v$ showed the highest solubilization potential produced the highest solubility of CXB $(3.044 \pm 0.0552 \mathrm{mg} / \mathrm{mL})$ compared to the other two cosolvents, PG and glycerol. Solubilization powers $(\sigma)$ of PEG 600, PG, and glycerol cosolvents were calculated by regression analysis from the log-linear solubilization plot and found to be $3.0563\left(R^{2}=0.9999\right), 2.8939\left(R^{2}=0.9955\right)$, and $1.4435\left(R^{2}=0.9853\right)$, respectively, since the cosolvents act by decreasing the density of the hydrogen bonds of water, thus creating a less-polar environment in the mixture that enhances the miscibility of drug molecules in the solution. As predicted, PEG 600 being less-polar exhibited the highest $(p<0.05)$ solubilization power compared to propylene glycol and glycerol. The chemical structure of PEG 600, $\mathrm{H}-\left[\mathrm{O}-\mathrm{CH}_{2}-\right.$ $\mathrm{CH} 2] \mathrm{n}-\mathrm{OH}$, facilitates its miscibility in water through the formation of hydrogen bonds. Moreover, PEG 600 has a high surface tension $\left(44.6 \mathrm{dyne} / \mathrm{cm}\right.$ at $20^{\circ} \mathrm{C}$ ) and wide dielectric constant ranges. With the help of the hydrophobic hydrocarbon region of $\mathrm{CXB}$, hydrogen bonds between water were broken, thus allowing the hydrophobic compounds to fit in [24].

3.3. Particle Size Analysis. The particle size was determined in a range between $0.1 \mathrm{~nm}$ and $10 \mu \mathrm{m}$ using a Malvern ${ }^{\circledR}$ Zetasizer. The mean particle size and polydispersity index (PDI) values for three samples prepared with PEG 600 at different concentrations 40,60 , and $80 \% v / v$ were $538.1 \mathrm{~nm} \pm 62.12$ (0.57), $256.1 \mathrm{~nm} \pm 6.652$ (1.00), and $235.3 \mathrm{~nm} \pm 16.42$ (0.16), respectively. The smallest particle size of CXB, a narrow size distribution, was achieved with $80 \%$ PEG solution indicating the maximum solubility of CXB (Figure 4). Knowing that the PDI value describes the degree of nonuniformity of the size distribution of particles within a sample, this index is dimensionless and scaled such that the PDI value preferred to fall in the range of $0.05-0.7$, which practically indicates a suitable degree of homogeneity of the sample [25].

3.4. Physical Appearance and pH of the In Situ Gel. Based on the obtained results, CXB solubilized in $80 \% v / v$ of PEG 600 in water was selected to prepare the in situ gel. All the prepared SA-based in situ gels of CXB were clear and 

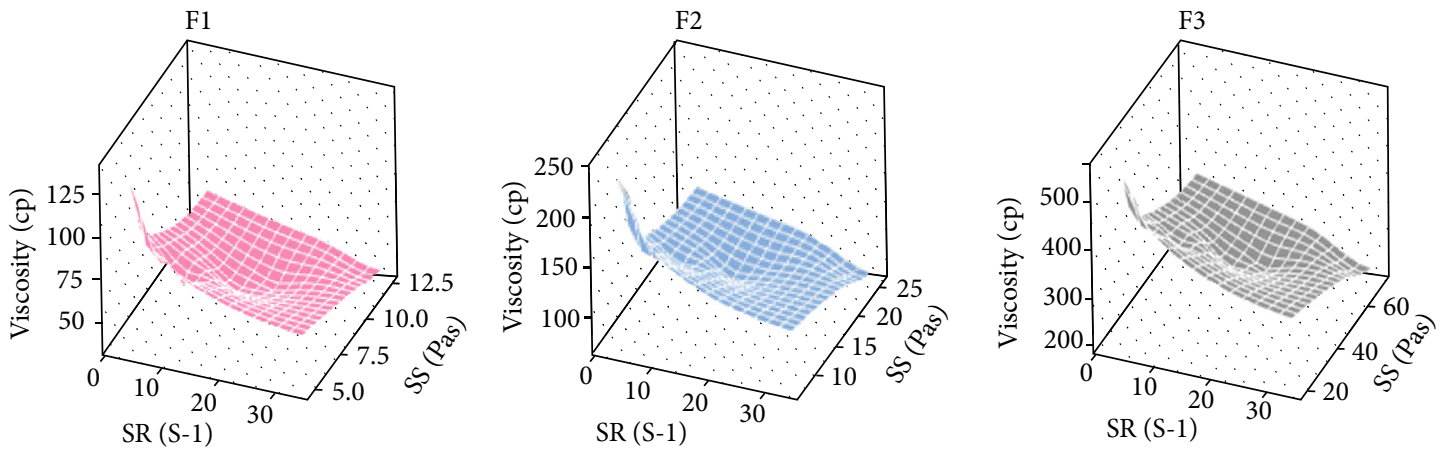

(a)

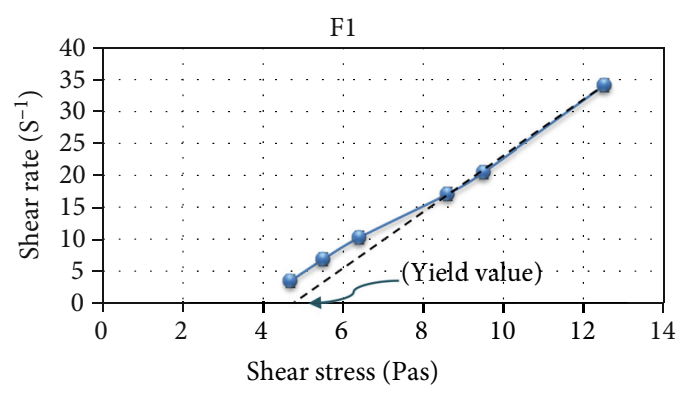

F2

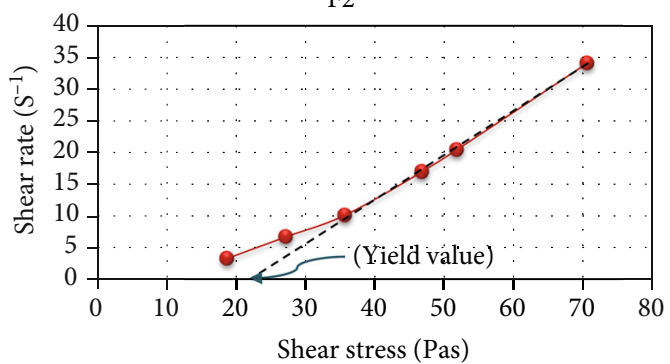

F3

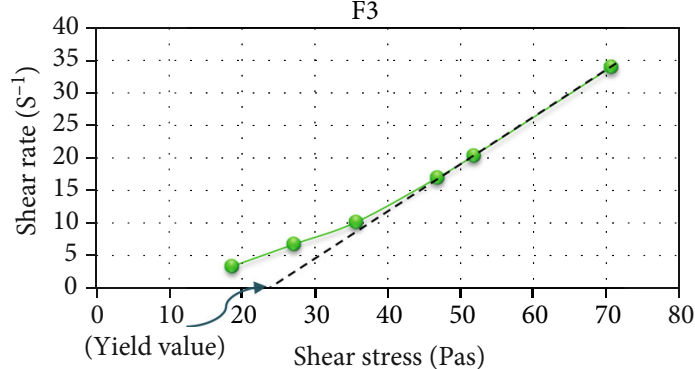

(b)

Figure 5: (a) 3D-surface plot of viscosity and shear stress as a function of shear rate for F1, F2, and F3. The graph was prepared by Minitab ${ }^{\circledR}$ 19. (b) Rheograms of the sol systems (F1, F2, and F3); the viscosity is given as a mean $\pm \mathrm{SD}(\mathrm{n}=3)$ at $25^{\circ} \mathrm{C}$.

homogenous. The mean $\mathrm{pH}$ values of the formulations in the form of the sol system were in the range of 5.63-6.13 (Table 3). A citrate buffer was added to the formulations to maintain their $\mathrm{pH}$ values relatively constant in the gastric medium. Consequently, it might ensure safety and compatibility of the formulations with the biological system in the form of an oral dosage form [26].

3.5. Rheological Behavior. Results revealed that viscosity of the prepared sols was directly related to the concentration of SA. The highest value of viscosity was shown by F3
$(562.5 \pm 17.68 \mathrm{cp})$ followed by F2 $(237.5 \pm 17.68 \mathrm{cp})$ and F1 $(137.5 \pm 17.68 \mathrm{cp})$, at a shear rate of $3.4 \mathrm{~S}^{-1}(10 \mathrm{rpm})$. However, increasing the shear rate to $34 \mathrm{~S}^{-1}(100 \mathrm{rpm})$ resulted in a significant $(p<0.05)$ decrease in the viscosity of all formulas (F3: $207.5 \pm 6.35 \mathrm{cp}, \mathrm{F} 2: 74 \pm 1.41 \mathrm{cp}$, and F1: 38.75 $\pm 1.77 \mathrm{cp}$ ) (Figure 5(a)). Viscoelastic fluids exhibit high viscosity under a low shear rate and vice versa. This kind of rheological behavior is more preferred in pharmaceutical preparations since it helps the easy administration of sol preparations at the site of administration followed by conversion into a gel structure in vivo which is desirable for release 


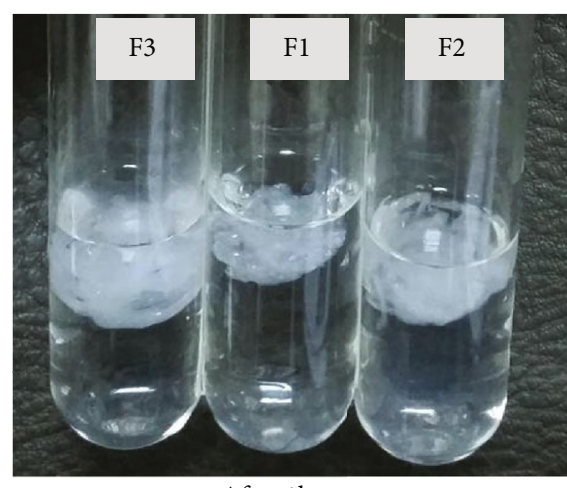

After $1 \mathrm{~h}$

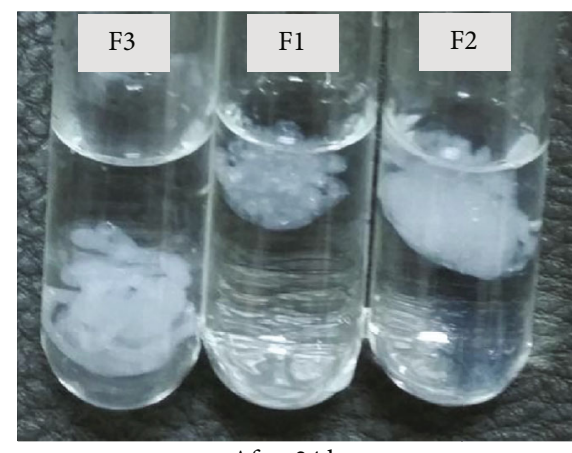

After 24 hs

Figure 6: Floating behaviors of F1, F2, and F3 in situ gels in the simulated gastric medium after $1 \mathrm{~h}$ and $24 \mathrm{hs}$.

sustainability of the drug [27]. Further data analysis was performed via the preparation of rheograms by plotting the shear rate $\left(\mathrm{S}^{-1}\right)$ vs. shear stress (Pas) to grasp the formulas' flow behavior. All formulas showed a non-Newtonian plastic rheological behavior where a certain amount of force must be applied to the fluid before the induction of any flow. This force is called the yield value. Once the yield value is exceeded and flow begins, plastic fluids may display a Newtonian flow. $\mathrm{F} 3$ presented the highest yield value comparing to F1 and F2 (Figure 5(b)). Moreover, all the prepared sols showed convenient flow properties and acceptable pourability, which is necessary to ensure ease of drug administration to the patient.

3.6. Floating Behavior. All the prepared formulas showed instant gelation upon contact with the simulated gastric fluid. The floating lag time varied with the formulation variables. F1 exhibited the least FLT (8.32 s), while F3 had the highest lag time $(22.5 \mathrm{~s})$. The fast buoyancy behavior of the formulas can be attributed to the presence of the floating agent, calcium carbonate, in the formulations as an insoluble dispersion. Hydrochloric acid, the key component of the gastric juice, reacted with calcium carbonate and formed $\mathrm{CaCl}_{2}$, water, and $\mathrm{CO}_{2}$ gas. $\mathrm{CaCl}_{2}$ is highly soluble in the aqueous solution $\left(74.5 \mathrm{~g} / 100 \mathrm{~mL}\right.$ at $\left.20^{\circ} \mathrm{C}\right)$ and is ionized in the solution to form $\mathrm{Ca}^{+2}$ and $\mathrm{Cl}^{-2}$ ions [28]. $\mathrm{Ca}^{+2}$ ions also ensued from the ionization of $\mathrm{CaCl}_{2}$, a cross-linking agent in the formulations. These ions were capable to cross-link SA molecules and form the double-helical three-dimensional

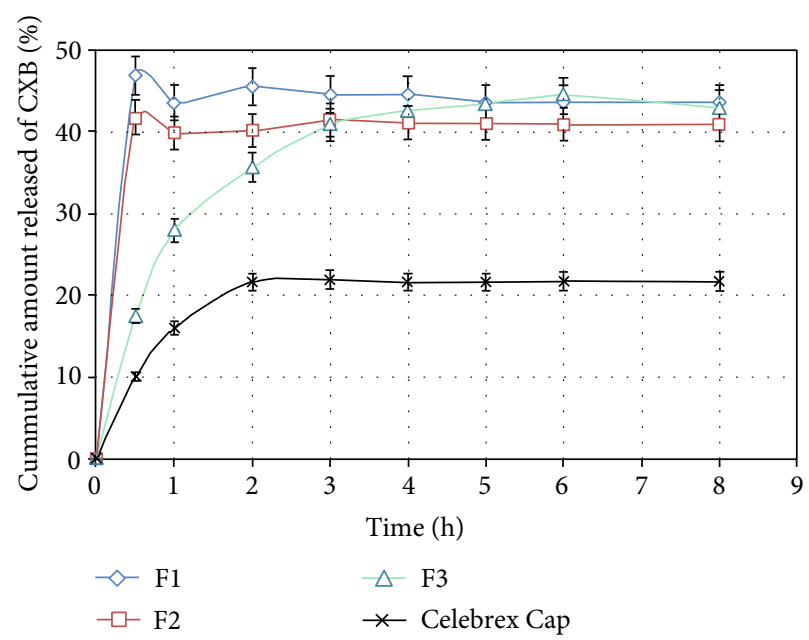

FIGURE 7: In vitro drug release from alginate-in situ gel formulations compared with the reference product (mean $\pm \mathrm{SD}, n=3$ ).

network structure of the gellious matrix, while the released $\mathrm{CO}_{2}$ gas got entrapped in the gel and resulted in floating of the matrix.

DOF of F3 was more than 12 hs but less than 24 hs, while $\mathrm{F} 1$ and F2 maintained the gel structure for longer than $24 \mathrm{~h}$ (Figure 6). The buoyancy behavior of F3, the longest FLT and shortest DOF, can be imputed to the increase in the SA content in the formulation, which consequently made the floating more difficult for the shortened time. However, all formulas presented good gelation capacity (Table 3), which is advantageous, since the drug sol is promptly converted into a thick gel matrix which floated over the stomach juice and worked as a reservoir releasing the drug in a sustained manner during the course of therapy [29]. In some previous studies, the gelation code $(+++)$ indicated that "gelation" was immediate and retained its integrity for an extended period of time; however, the gel was stiff which may cause irritation and discomfort to the patient while $(++)$ demonstrated the optimum gelation characteristics $[30,31]$.

3.7. In Vitro Drug Release. The amount of CXB released in the dissolution medium was determined from the regression line equation, $y=84.132 x-0.0068\left(R^{2}=0.9994\right)$, which was obtained from the previously prepared calibration curve of $\mathrm{CXB}$ in $0.1 \mathrm{~N} \mathrm{HCl}$ at $\lambda_{\max } 233$. The concentration of SA was found to be essentially affecting the pattern of the drug's release. The cumulative amount released by CXB significantly $(p<0.05)$ decreased with the increment of the polymer's concentration. This could be due to either the entrapment of CXB inside the networks of the gel's matrix or the polymer-drug molecular interaction. Similar results were also reported in previously published research works [32]. Despite F1 releasing the highest amount of CXB during the first half an hour of the test compared to F2 and F3, the optimum sustained drug release profile till the end of the run was practiced by F3, as shown in Figure 7. Moreover, the amount of CXB released from all formulas was significantly $(p<0.05)$ much higher than that of the reference drug (Celebrex ${ }^{\circledR}$ capsule by Pfizer, dose: $200 \mathrm{mg}$ of CXB). This 
TABLE 4: Results of curve fitting of the in vitro diclofenac sodium release data from different optimized alginate-PVP K30 microbeads.

\begin{tabular}{|c|c|c|c|c|c|c|c|c|c|}
\hline \multirow{2}{*}{ Formula code } & \multicolumn{2}{|c|}{ Zero-order kinetics } & \multicolumn{2}{|c|}{ First-order kinetics } & \multirow{2}{*}{$\begin{array}{c}\text { Higuchi model } \\
K_{H}\left(\% \mathrm{~h}^{-1 / 2}\right)\end{array}$} & \multicolumn{4}{|c|}{ Korsmeyer-Peppas model } \\
\hline & $K_{\mathrm{o}}\left(\% \mathrm{~h}^{-1}\right)$ & $R^{2}$ & $K_{1}\left(\mathrm{~h}^{-1}\right)$ & $R^{2}$ & & $R^{2}$ & $K_{\mathrm{KP}}\left(\% \mathrm{~h}^{-1}\right)^{2}$ & $n$ & $R^{2}$ \\
\hline $\mathrm{F} 1$ & 4.456 & 0.625 & 0.097 & 0.4286 & 24.116 & 0.6332 & 45.245 & 0.216 & 0.505 \\
\hline $\mathrm{F} 2$ & 2.547 & 0.167 & 0.086 & 0.019 & 24.087 & 0.6536 & 41.264 & 0.325 & 0.601 \\
\hline F3 & 2.308 & 0.209 & 0.027 & 0.5793 & 22.105 & 0.9877 & 25.687 & 0.494 & 0.886 \\
\hline
\end{tabular}

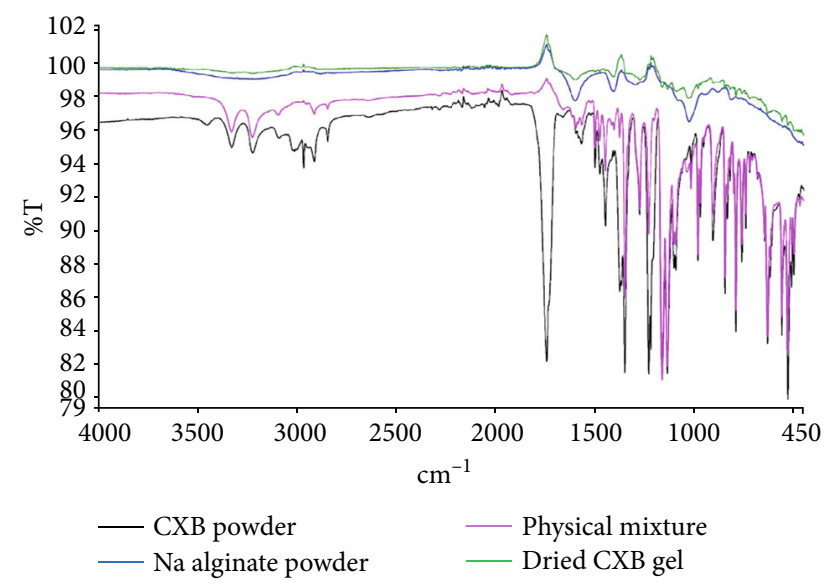

FIgURE 8: FTIR spectra of CXB powder, SA powder, physical mixture, and $\mathrm{CXB}$-dried gel. Color legend indicates the following: black: CXB; blue: SA; pink: CXB/SA physical mixture; and green: CXB-dried gel.

improvement in drug release can be attributed to the efficiency of the used technique, cosolvency, for CXB solubilization.

The kinetics modeling study on in vitro drug release revealed that the Higuchi model was superior in describing the in vitro release of $\mathrm{CXB}$ from the optimized formulation, F3, as shown in Table 4. In addition, the mechanism of the drug's release for all formulas was found to be a Fickian diffusion release since the $n$ value of all formulas was $\leq 0.5$ [33]. Thus, the flux of CXB from the delivery system to the dissolution medium is basically controlled by the gel's matrix's thickness and the concentration gradient across a specified sectional area.

3.8. FTIR Spectroscopy. Figure 8 represents the FTIR spectra of $\mathrm{CXB}, \mathrm{SA}, \mathrm{CXB} / \mathrm{SA}$ physical mixture, and CXB dried gel. In IR spectra, CXB showed medium absorption bands at $3229 \mathrm{~cm}^{-1}$ and $3334 \mathrm{~cm}^{-1}$, which were assigned to the drug-NH symmetric and asymmetric stretching vibrations of the primary amine group. The other characteristic bands may be attributed to the following group vibrations: a strong peak at $1738 \mathrm{~cm}^{-1}(\mathrm{~N}-\mathrm{H}$ bending), 1445 and $1497 \mathrm{~cm}^{-1}$ ( $\mathrm{S}=\mathrm{O}$ symmetric and asymmetric stretching, respectively), and $791 \mathrm{~cm}^{-1}$ (aromatic $-\mathrm{CH}$ bend). The FTIR spectra of SA pure powder showed stretching vibrations of the $\mathrm{O}-\mathrm{H}$ functional group at wavenumbers $3200-$ $3400 \mathrm{~cm}^{-1}$, and peaks at $1404 \mathrm{~cm}^{-1}$ and $1597 \mathrm{~cm}^{-1}$ were attributed to asymmetric and symmetric stretching vibrations of carboxylate salt ion, respectively, in addition to the mannuronic acid functional group at wavenumber $884 \mathrm{~cm}^{-1}$ and the uronic acid at wavenumber $1025 \mathrm{~cm}^{-1}$.

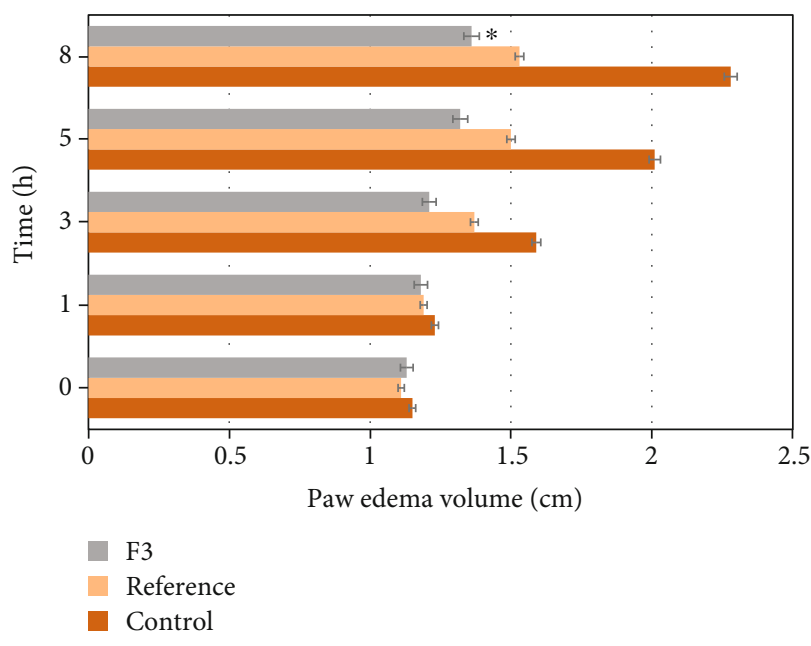

Figure 9: Effect of CXB on carrageenan-induced rat paw edema at different time intervals. Results are given as mean \pm SEM $(n=6)$, ${ }^{*}$ Significant effect at $p<0.05$.

In the IR spectrum of the CXB/SA physical mixture, $\mathrm{CXB}$ peaks were prominent than $\mathrm{SA}$, which seems superimposed by $\mathrm{CXB}$. Moreover, the spectrum showed the absence of major peaks, the $\mathrm{N}-\mathrm{H}$ bending vibration $\left(1738 \mathrm{~cm}^{-1}\right)$ of the amine functional group and the $\mathrm{O}-\mathrm{H}$ stretching vibrations $\left(3200-3400 \mathrm{~cm}^{-1}\right)$ of the carboxylic group, which were present in the spectrum of CXB and SA powders alone, respectively. On the other hand, changes in the spectrum of the gel sample were more noticeable than in the physical mixture (Figure 8). There was a decrease in the intensity of carboxylate peaks at $1404 \mathrm{~cm}^{-1}$ and $1597 \mathrm{~cm}^{-1}$ in the gel spectrum compared to the pure component, which could be due to alginate cross-linking by calcium ions to form the egg-box structure of the gel [34]. The spectrum exhibited changes in the peaks of both $\mathrm{CXB}$ and SA, confirming the molecular interaction of $\mathrm{CXB}$ with SA in the gel. These findings strongly supported the idea of intermolecular hydrogen-bonding between the $\mathrm{C}=\mathrm{O}$ group of $\mathrm{SA}$ and the $\left(-\mathrm{NH}_{2}\right)$ group of the sulfonamide substituent in the $\mathrm{CXB}$ molecule. Hydrogen bonding between SA and chemical compounds was also reported in previous studies $[35,36]$. On the contrary, the ionic drug-polymer interaction had unlikely formed, as $\mathrm{CXB}$ is a weak organic acid $(\mathrm{pKa}=11.1)$ that predominantly exists in the unionized form at $\mathrm{pH}$ values below its $\mathrm{pKa}$.

\subsection{In Vivo Evaluation in Animals}

3.9.1. Anti-Inflammatory Activity. The optimized floating in situ gel formula of CXB (F3) has shown significant inhibition of carrageenan-induced rat paw edema from 2 to $8 \mathrm{hrs}$ in rats 
TABLE 5: Anti-inflammatory effect of F3, reference, and control in carrageenan-induced paw edema in rats.

\begin{tabular}{lccccccc}
\hline \multirow{2}{*}{ Group } & \multirow{2}{*}{ Treatment } & \multirow{2}{*}{ Initial paw volume } & \multicolumn{4}{c}{ Paw volume (mL) } & \multicolumn{2}{c}{ Edema inhibition at 8 hs (\%) } \\
\hline I & Control & $1.15 \pm 0.07$ & $1.23 \pm 0.02$ & $1.59 \pm 0.08$ & $2.01 \pm 0.03$ & $2.28 \pm 0.06$ & - \\
II & Reference & $1.11 \pm 0.08$ & $1.19 \pm 0.07$ & $1.37 \pm 0.05^{*}$ & $1.50 \pm 0.05^{*}$ & $1.53 \pm 0.01^{*}$ & 75.83 \\
III & F3 & $1.13 \pm 0.05$ & $1.18 \pm 0.04$ & $1.21 \pm 0.02^{*}$ & $1.32 \pm 0.03^{*}$ & $1.36 \pm 0.04^{*}$ & 92.64 \\
\hline
\end{tabular}

Values are represented as mean \pm SEM, $n=6$ in each group, two-way ANOVA followed by Bonferroni’s multiple comparisons, ${ }^{*} p<0.05$.

TABLE 6: The analgesic activity on rats measured by latency period (s) using the hot plate method.

\begin{tabular}{lcccc}
\hline & \multicolumn{4}{c}{ Latency $(\mathrm{sec})$} \\
Time $(\mathrm{h})$ & $0 \mathrm{~h}$ & $0.5 \mathrm{~h}$ & $1 \mathrm{~h}$ & $3 \mathrm{~h}$ \\
\hline Control & $16.10 \pm 3.36$ & $16.75 \pm 2.62$ & $16.75 \pm 3.2$ & $16.10 \pm 1.36$ \\
Reference & $15.52 \pm 1.00$ & $23.12 \pm 1.63$ & $25.75 \pm 1.7^{*}$ & $21.75 \pm 1.95$ \\
F3 & $15.75 \pm 0.95$ & $30.25 \pm 3.59^{* *}$ & $36.50 \pm 8.8^{* *}$ & $37.5 \pm 2.40^{* *}$ \\
\hline
\end{tabular}

Values are represented as mean $\pm \mathrm{SEM}, n=6$ in each group, two-way ANOVA followed by Bonferroni's multiple comparisons, ${ }^{*} p<0.05$ and ${ }^{* *} p<0.001$.

following oral administration, as compared with the control and standard group of animals (Figure 9). The highest percentage of inhibition of the standard drug was found to be $75.83 \%(p<0.05)$, whereas the optimized floating in situ CXB gel formula (F3) has $92.64 \%$ inhibition of paw edema at $8 \mathrm{~h}(p<0.05)$, as shown in Table 5. Although the standard drug showed significant inhibition of paw edema, the optimized floating in situ gel showed a higher percentage of inhibition at $8 \mathrm{~h}$.

3.9.2. Analgesic Activity. The results of the analgesic activity test are represented in Table 6. Formulation F3 showed high and persistent analgesic activity starting from $0.5 \mathrm{~h}$ till the end of the 3 hours $(p<0.001)$ compared to the reference drug that showed the maximum activity after one hour $(p<0.05)$. F3 formulation, with a sustained release pattern, ensured the prolonged duration of analgesia that persists for the entire experimental time.

\section{Conclusion}

The present study evaluated and compared the aqueous solubility enhancement of CXB using three different cosolvents, namely, polyethylene glycol (PEG 600), propylene glycol (PG), and glycerin. PEG $60080 \% v / v$ was selected in terms of the most efficient solubilizing cosolvent and particle size distribution. The CXB formulated as a floating in situ gel containing $1 \% \mathrm{SA}$ is a potential candidate for the oral administration of $\mathrm{CXB}$ that can provide a sustained release pattern with Higuchi model kinetics. FTIR spectroscopy analysis proved a considerable interaction between CXB and SA molecules, which played a significant role in modifying the drug release from the gel delivery system. The candidate formulation F3 showed high and persistent analgesic and antiinflammatory activities in rats compared to the commercial reference product.

\section{Data Availability}

The data used to support the findings of this study are included within the article.

\section{Conflicts of Interest}

The authors declare no potential conflict of interest.

\section{Acknowledgments}

We would like to thank Dr. Ramachandran Subramanian, Associate Professor and Head of Biotechnology Department, Birla Institute of Technology and Science, Pilani, Dubai Campus, United Arab Emirates, for his kind help to perform the particle size test; we also thank fourth-year students for their assistance in the lab work. This research work was funded by the Dubai Pharmacy College, Dubai, UAE.

\section{References}

[1] M. M. Goldenberg, "Celecoxib, a selective cyclooxygenase-2 inhibitor for the treatment of rheumatoid arthritis and osteoarthritis," Clinical Therapeutics, vol. 21, no. 9, pp. 1497-1513, 1999.

[2] S. K. Paulson, M. B. Vaughn, S. M. Jessen et al., "Pharmacokinetics of celecoxib after oral administration in dogs and humans: effect of food and site of absorption," Journal of Pharmacology and Experimental Therapeutics, vol. 297, no. 2, pp. 638-645, 2001.

[3] US Food and Drug Administration, The Biopharmaceutics Classification System (BCS) guidance, US Food and Drug Administration - Center for Drug Evaluation and Research, 2019.

[4] W. H. Song, D. W. Yeom, D. H. Lee et al., "In situ intestinal permeability and in vivo oral bioavailability of celecoxib in supersaturating self-emulsifying drug delivery system," Archives of Pharmacal Research, vol. 37, no. 5, pp. 626-635, 2014.

[5] Z. S. Eren, S. Tunçer, G. Gezer, L. T. Yildirim, S. Banerjee, and A. Yilmaz, "Improved solubility of celecoxib by inclusion in SBA-15 mesoporous silica: Drug loading in different solvents and release," Microporous and Mesoporous Materials, vol. 235, pp. 211-223, 2016.

[6] H. I. Kim, S. Park, S. Park et al., "Development and evaluation of a reconstitutable dry suspension to improve the dissolution and oral absorption of poorly water-soluble celecoxib," Pharmaceutics, vol. 10, no. 3, p. 140, 2018.

[7] H.-I. Kim, J.-P. Jee, S. T. Kim et al., "Preparation and characterization of celecoxib nanosuspension using bead milling," 
Journal of Nanoscience and Nanotechnology, vol. 19, no. 2, pp. 1184-1187, 2019.

[8] K. H. Cho, J.-P. Jee, D. A. Yang et al., "Improved dissolution and oral bioavailability of celecoxib by a dry elixir system," Journal of Nanoscience and Nanotechnology, vol. 18, no. 2, pp. 1482-1486, 2018.

[9] E.-S. Ha, G.-H. Choo, I.-H. Baek, and M.-S. Kim, "Formulation, characterization, and in vivo evaluation of celecoxibPVP solid dispersion nanoparticles using supercritical antisolvent process," Molecules, vol. 19, no. 12, pp. 20325-20339, 2014.

[10] L. Kagan and A. Hoffman, "Selection of drug candidates for gastroretentive dosage forms: pharmacokinetics following continuous intragastric mode of administration in a rat model," European Journal of Pharmaceutics and Biopharmaceutics, vol. 69, no. 1, pp. 238-246, 2008.

[11] H. B. Nirmal, S. R. Bakliwal, and S. P. Pawar, "In-situ gel: new trends in controlled and sustained drug delivery system," International Journal of PharmTech Research, vol. 2, no. 2, pp. 1398-1408, 2010.

[12] R. Bashir, A. Majeed, T. Ali, S. Farooq, and N. A. Khan, "Floating oral in-situ gel: a review," Journal of Drug Delivery and Therapeutics, vol. 9, no. 2, pp. 442-448, 2019.

[13] M. Madan, A. Bajaj, S. Lewis, N. Udupa, and J. A. Baig, "In situ forming polymeric drug delivery systems," Indian Journal of Pharmaceutical Sciences, vol. 71, no. 3, pp. 242-251, 2009.

[14] S. R. Karajgi, S. Metri, V. Tiwari, S. Hulyalkar, T. A. Rub, and A. S. Patil, "UV spectrophotometric method for the quantitative estimation of celecoxib in capsule dosage forms," Der Pharmacia Lettre, vol. 8, no. 10, pp. 247-257, 2016.

[15] E. Baka, J. E. A. Comer, and K. Takács-Novák, "Study of equilibrium solubility measurement by saturation shake-flask method using hydrochlorothiazide as model compound," Journal of Pharmaceutical and Biomedical Analysis, vol. 46, no. 2, pp. 335-341, 2008.

[16] M. I. Tadros, "Controlled-release effervescent floating matrix tablets of ciprofloxacin hydrochloride: development, optimization and in vitro-in vivo evaluation in healthy human volunteers," European Journal of Pharmaceutics and Biopharmaceutics, vol. 74, no. 2, pp. 332-339, 2010.

[17] N. Yuksel, A. E. Kanik, and T. Baykara, "Comparison of in vitro dissolution profiles by ANOVA-based, modeldependent and -independent methods," International Journal of Pharmaceutics, vol. 209, no. 1-2, pp. 57-67, 2000.

[18] Committee for the Update of the Guide for the Care and Use of Laboratory Animals, Institute for Laboratory Animal Research, Division on Earth and Life Studies, National Research Council, Guide for the care and use of laboratory animals, The National Academies Press, 2011.

[19] C. A. Winter, E. A. Risley, and G. W. Nuss, "CarrageeninInduced edema in hind Paw of the rat as an assay for Antiinflammatory drugs," Experimental Biology and Medicine, vol. 111, no. 3, pp. 544-547, 1962.

[20] B. K. A. Rasool, E. Abu-Gharbieh, S. Fahmy, H. Saad, and S. Khan, "Development and evaluation of ibuprofen transdermal gel formulations," Tropical Journal of Pharmaceutical Research, vol. 9, no. 4, pp. 355-363, 2010.

[21] N. Seedher and S. Bhatia, "Solubility enhancement of cox-2 inhibitors using various solvent systems," AAPS Pharm Sci Tech, vol. 4, no. 3, pp. 36-44, 2003.
[22] S. H. Yalkowsky and J. T. Rubino, "Solubilization by cosolvents I: organic solutes in propylene glycol-water mixtures," Journal of Pharmaceutical Sciences, vol. 74, no. 4, pp. 416-421, 1985.

[23] Y. Miyako, Y. Zhao, K. Takeshima, T. Kataoka, T. Handa, and R. Pinal, "Solubility of hydrophobic compounds in watercosolvent mixtures: relation of solubility with watercosolvent interactions," Journal of Pharmaceutical Sciences, vol. 99, no. 1, pp. 293-302, 2010.

[24] A. K. Nayak and P. P. Panigrahi, "Solubility enhancement of etoricoxib by cosolvency approach," ISRN Physical Chemistry, vol. 2012, 5 pages, 2012.

[25] U. Nobbmann, "Polydispersity - what does it mean for DLS and chromatography?," Malvern PanalyticalOctober 2017, https:// www.materials-talks.com/blog/2017/10/23/polydispersitywhat-does-it-mean-for-dls-and-chromatography/.

[26] P. Khulbe, B. Shrivastava, P. Sharma, and A. K. Tiwari, "In-situ buffered formulation: an effective approach for acid labile drug," International Journal of Pharmaceutical Sciences and Research, vol. 8, no. 1, pp. 35-44, 2017.

[27] M. Abhirami, R. Brindha, S. Gomathi, B. N. Vedha Hari, and D. Ramya Devi, "Optimization of in-situ gelling system for nasal administration of celecoxib," Journal of Chemical and Pharmaceutical Research, vol. 6, pp. 502-515, 2014.

[28] Drug Bank, Calcium chloride, 2019, https://www.drugbank.ca/ drugs/DB01164.

[29] H. Xu, M. Shi, Y. Liu, J. Jiang, and T. Ma, “A novel in situ gel formulation of ranitidine for oral sustained delivery," Biomolecules \& Therapeutics, vol. 22, no. 2, pp. 161-165, 2014.

[30] J. Balasubramaniam, S. Kant, and J. K. Pandit, "In vitro and in vivo evaluation of the Gelrite gellan gum-based ocular delivery system for indomethacin," Acta Pharmaceutica, vol. 53, no. 4, pp. 251-261, 2003.

[31] N. M. Harish, P. Prabhu, R. N. Charyulu, M. A. Gulzar, and E. V. S. Subrahmanyam, "Formulation and evaluation of in situ gels containing clotrimazole for oral candidiasis," Indian Journal of Pharmaceutical Sciences, vol. 71, no. 4, pp. 421427, 2009.

[32] A. Nayak, S. Khatua, M. Hasnain, and K. Sen, "Development of diclofenac sodium-loaded alginate-PVP K 30 microbeads using central composite design," Daru, vol. 19, no. 5, pp. 356-366, 2011.

[33] P. L. Ritger and N. A. Peppas, "A simple equation for description of solute release I. Fickian and non-Fickian release from non-swellable devices in the form of slabs, spheres, cylinders or discs," Journal of Controlled Release, vol. 5, no. 1, pp. 2336, 1987.

[34] B. Mandal, K. S. Alexander, and A. T. Riga, "Evaluation of the drug-polymer interaction in calcium alginate beads containing diflunisal," Pharmazie, vol. 65, no. 2, pp. 106-109, 2010.

[35] L. Yang, J. Guo, Y. Yu et al., "Hydrogen bonds of sodium alginate/Antarctic krill protein composite material," Carbohydrate Polymers, vol. 142, pp. 275-281, 2016.

[36] S. R. Derkach, N. G. Voron'ko, N. I. Sokolan, D. S. Kolotova, and Y. A. Kuchina, "Interactions between gelatin and sodium alginate: UV and FTIR studies," Journal of Dispersion Science and Technology, vol. 41, no. 5, pp. 690-698, 2020. 